\title{
Analysis and Matching of Composite Sketches to Facial Photograph Using Component Based Approaches
}

\author{
P. Mohod ${ }^{1}$ and S .Khandait ${ }^{2}$ \\ ${ }^{1}$ GHRCE, Nagpur, Maharashtra \\ ${ }^{2}$ Department of Information Technolgy, KDK.College of Engg.,Nagpur, MH \\ \{psmohod@gmail.com prapti_khandait@yahoo.co.in\}
}

\begin{abstract}
Facial Sketches are most widely used in law enforcement agencies for identification and apprehension of suspects, which may involve in several criminal activities. If the photographs of the suspect is stored in the mug shot data base, then the identity of the suspect is possible, but in most of the cases that it is a possibility that the photographs of the suspect is not available then two methods are used first is forensic sketch and the second is composite sketch software. The forensic sketch which is drawn by the expert artist according to verbal description provided by an eyewitness Unlike sketches, hand drawn by artist, composite sketches are drawn using one of the several facial composite software system (FACES and Mac-a-Mug etc). We propose a component based representation (CBR) approach that is used to measure the similarity between composite sketches and facial photographs. First the preprocessing technique is applied on both sketches as well as on face photo and after that components are extracted using 13 point marking and after that the multi scale local binary pattern (MLBP) is applied on each of the extracted region of face photo and sketch and finally the similarity which is obtained from individual facial photograph and sketch components are fused together so, that the total components similarity is obtained. Experimental results for matching 100 composites against 100 facial photographs show that the proposed method achieves (56\% to $70 \%$ ) performance as compared to a leading commercial face recognition system. We believe that our prototype system will be of great value in law enforcement agencies for catching a flitting suspect in a timely fashion
\end{abstract}

\section{Keywords: LBP ASM PCA.}

\section{Introduction}

Face recognition is a very important application of criminal's identity which is involved in various types of criminal activity. If a facial photographs of suspects are available, then the identity of the criminal is possible, but in most of the cases there is a possibility that the images of the suspect is not available in a mug shot, then in that case a Forensic method will be applied, in forensic method the sketches were drawn by expert artist with the help of verbal description which is to be provided by the eyewitness. Due to budgetary reasons, many law enforcement agencies use facial composite software, which allows the user to create composite sketches (computer generated facial composite) instead of using hand drawn forensic sketches. The composite sketches are better than forensic because facial composite software kits, which allow even a non-artist to draw a sketch after only several hours of training. Some of the widely used facial composite software kits include Identikit, Photo-Fit, FACES, Mac-a-Mug, and EvoFIT. The $80 \%$ of the officers in the law enforcement agencies used composite sketches. Despite of this high percentage of law enforcement agencies using a computer generated composites, the automated face recognition for computer generated composites has not been studied. In this paper, we propose a face recognition system to match composite sketches to facial photographs or mug shot.

To do this task, we propose a new face recognition algorithm for matching composite sketches to facial photographs. The objective of this approach is to measure the similarity between sketches

B. Iyer, S. Nalbalwar and R.Pawade (Eds.)

ICCASP/ICMMD-2016. Advances in Intelligent Systems Research.

Vol. 137, Pp. 506-513.

(C) 2017- The authors. Published by Atlantis Press

This is an open access article under the CC BY-NC license (http://creativecommons.org/licens)es/by-nc/4.) 
and photographs. To effectively match composite sketches against a large scale gallery database. So that it can successfully recognize a face based on sketches.

The rest of this paper is structured as follows section II is introduces the related work (literature survey) on face recognition. In section III the proposed component based representation (CBR) for composite sketch recognition is present. In section IV, experiments are performed to verify the effectiveness of CBR approaches for matching purposes. Finally, Section V includes our contributions and the lists the directions of future work.

\section{Related Work on Face Recognition}

\subsection{Criminal Identification in color skin images: Using Skin marks [1]}

Relatively Permanent Pigmented or Vascular Skin Marks (RPPVSM) were recently introduced as a biometric trait for identification in cases where the evidence images show only the non-facial body parts of the criminals or victims, such as in child sexual abuse and riots. As manual RPPVSM identification is tiring and time consuming, an automated RPPVSM identification system is proposed in this paper. The system is comprised of skin segmentation, RPPVSM detection, and RPPVSM matching algorithms.

\subsection{Low Resolution Androgenic pattern for Criminal and Victim Identification [2]}

Identifying criminals and victims in images (e.g., child pornography and masked gunmen) can be a challenging task, especially when neither their faces nor tattoos are observable. Skin mark patterns and blood vessel patterns are recently proposed to address this problem. However, they are invisible in low-resolution images and dense androgenic hair can cover them completely. Medical research results have implied that androgenic hair patterns are a stable biometric trait and have potential to overcome the weaknesses of skin mark patterns and blood vessel patterns. To the best of our knowledge, no one has studied androgenic hair patterns for criminal and victim identification before. This paper aims to study matching performance of androgenic hair patterns in low resolution images. An algorithm designed for this paper uses Gabor filters to compute orientation fields of androgenic hair patterns, histograms on a dynamic grid system to describe their local orientation fields, and the blockwise Chi-square distance to measure the dissimilarity between two patterns.[2]

\subsection{Face recognition using various classifiers}

Face recognition using artificial neural network, linear discriminant analysis, principal component analysis. Classifiers are used to increase the generalization accuracy for face recognition. (LDA), principal component analysis (PCA) etc. Since the classifier are very important in face recognition because it will take a definitive decision about the class and require training sample, all this is having only ' $\mathrm{P}$ ' dimensions So, with the help of PCA compression is possible [3]. 


\subsection{Locating facial feature with an extended active shape model [6]}

In this paper author proposed an extension active shape model by considering a more facial landmark. The extended active shape model is consisting of following extensions. 1) More landmark are consider then that of actually needed. 2) Choosing a two dimensional landmark instead of one landmark template 3) Noise to be added in training set. By considering the entire above factor which help the ASM [3] (Active Shape Model) to locate the facial feature so, it helps to identify the face photo of the suspect.

\subsection{Hand drawn face sketch recognition by human and PCA}

In this paper they have proposed a comparison performance of human and PCA (principal component analysis) in recognizing a facial sketch. There are five sketch artist since the sketch is drawn manually according to the description and each expert artist draw 50 sketches per subject so, for one subject there is about 250 sketches and also the fusion of sketches which is drawn by the different artist so, the recognition accuracy is improved. The main working of PCA is as follows : 1) $n$ dimensional is a original data 2) calculate ' $n$ ' Eigen vectors and Eigen values 3 ) choose only the first ' $p$ ' eigenvectors, based on their Eigen values 4) so, final data set is having only 'p' dimensions. So, with the help of PCA data compression is possible. [7]

\subsection{Matching forensic sketches to mug shot photos}

In this paper they have proposed matching a forensic sketch to mug shot data base. They have used a LFDA (local feature based discriminant analysis) for individually representation of both sketch and photo for this they have used a SIFT (Scale invariant feature transformation) and MLBP (multi scale local binary pattern) both are used as a feature descriptors with the help of LFDA matching is performed so that it was recognize the heterogeneous face based on sketches [5].

\subsection{EUCLBP matching algorithm for forensic application}

In this paper they have consider a three different types of sketches that are used for performance evolution: 1) Viewed sketch. 2) Semi forensic sketch 3) Forensic In this paper they have proposed a EUCLBP algorithm (extended uniform circular local binary pattern). The EUCLBP algorithm is applied to extract the facial information in a local facial region along with the minute details since both the sketch and facial image are divided into multi resolution pyramid, which help to build the discriminating the facial pattern that pattern is used to form a unique signature of both facial image and sketches. After that the memetic algorithm is applied to match this two EUCLBP descriptor. The LBP (local binary pattern) is used as a dense descriptor which helps to compute the texture feature for every pixel of an input image. The CLBP (circular local binary pattern) is corresponding to the gray level intensity of center pixel of circle. Since the optimized weight for the feature extracted image and matching that feature extracted image with the dataset so, that the top matches are obtain. [4] 


\section{Proposed Scheme}

Most of the law enforcement agencies are essentially used facial composite sketches which are drawn by the composite software system. A composite sketch is constructed by individually selecting a each facial components following a verbal description provided with an eyewitness. [3] Inspired by the principle of facial composite systems and existing work on photo to photo matching, we proposed a component based representation (CBR) for matching composite sketches to facial photograph. As illustrated in Figure 1

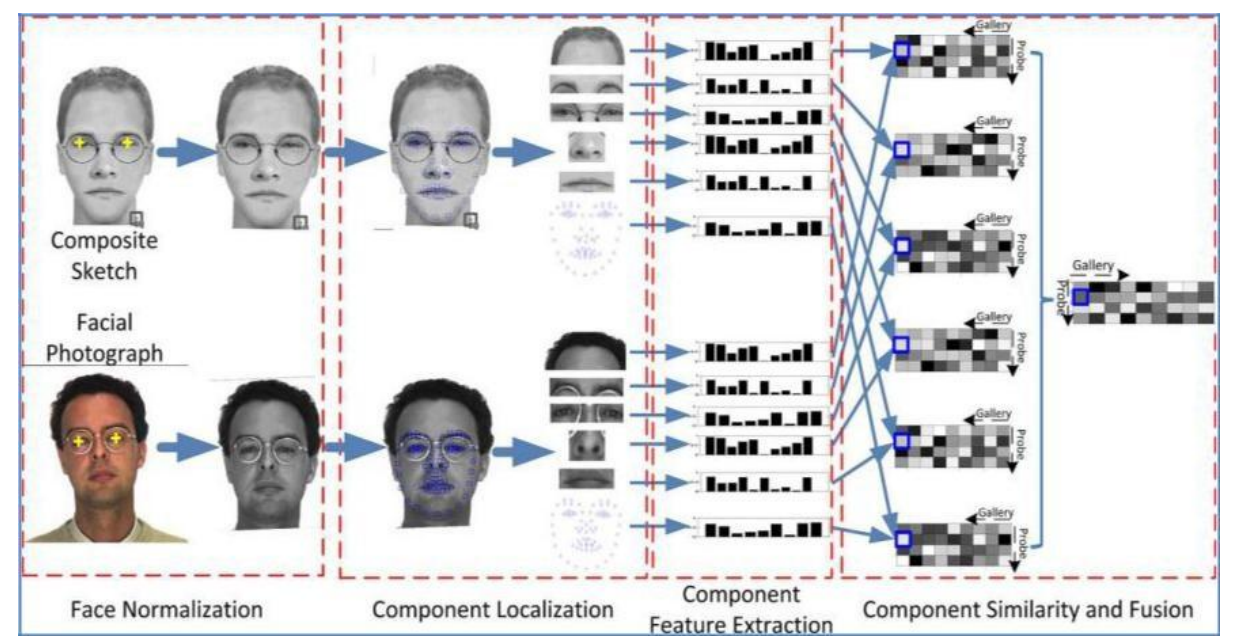

Figure 1: Overview of the Component-based representation approach for matching Composite sketches to face photos.

The component based representation (CBR) is having a four process. 1) Face normalization 2) Facial feature are extracted by considering a $12 * 12$ window with $60 \%$ overlapping.3) local feature is extracted with the help of LBP (local binary pattern) and LBP(multiscale local binary pattern) [6] 5) The last process consists of the matching process in which the histogram similarity is matched. The details of all these steps are explained as follows. [3]

\subsection{Face Normalization}

The face normalization are includes the preprocessing technique in which first the color conversion is performed on both sketches as well as on facial photographs so that both are converted into a gray level image. After that the Rotation is applied to converting gray level image and finally the Scaling is performed on Rotated image. Here, the dimension of the sketch is large as compared to facial photograph so, we have to fixed an image with the dimension of $200 * 150$ so, that the dimension of the sketch and facial photographs should not be varied. [1]

\subsection{Facial Component Localization}

The mug shot photographs are usually captured with the cooperation of the subject, and the composite sketches are typically synthesized in frontal pose. The pose and scale variations between facial photographs and composite sketches can be effectively eliminated with the above mentioned face normalization step. Therefore, in the proposed method, a more efficient approach is utilized 
for locating the facial components from normalized frontal photographs and composite sketches. Here, we are considering a window or component of $12 * 12$ with $60 \%$ overlapping and having an orientation of 8 pixels. Every time a $12 * 12$ window is considered with respect to the horizontal as well as vertical axes.[3]

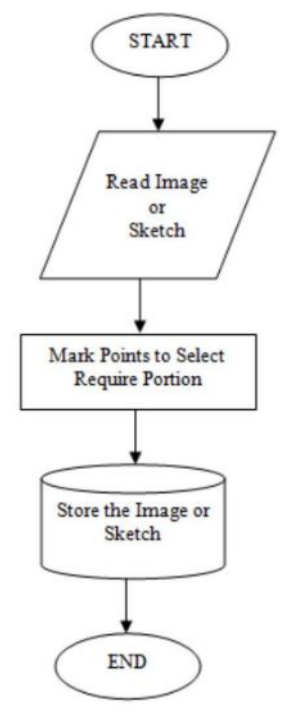

Figure 2: The flowchart for cutting an image and sketch

\subsection{Components Feature Extraction}

The feature which are extracted by considering a $12 * 12$ window with $60 \%$ overlapping, in each of the extracted component local binary pattern(LBP) is applied, which allow to robustly match corresponding facial components under the intensity discrepancy between composite sketches and facial photographs.

The LBP code of pixel is calculated by thresholding neighboring pixel intensity with the intensity of the center pixel. The LBP code of pixel $(\mathrm{x}, \mathrm{y})$ in image I can be calculated as

$$
L B P_{P}, R(x, y)=\sum_{p=0}^{p-1} 2^{p} \operatorname{sign}(I(x p, y p)-I(x, y))
$$

Where, $(x p, y p)$ is a $\mathrm{P}$ sampled neighbor pixel of pixel $(x, y)$, the distance from $(x p, y p)$ to $(x, y)$ is not larger than R pixel and Sign(.) function is defined as $\operatorname{Sign}(d)==\{1$ if $\mathrm{d}>0$ and 0 otherwise $\}$ but at some scale LBP is insufficient to capture all the features

\subsection{Components Similarity and Fusion}

After the decomposition of overlapped block, a facial component is divided into n patches. Let Ai, $\mathrm{Bi}$ be the photo patch and corresponding composite patch respectively, with MLBP (Multi Scale Local Binary Pattern), the feature extracted for $\mathrm{Ai}, \mathrm{Bi}$ is calculated based on the normalized histogram intersection. Now $\mathrm{Si}$ is calculated with the help of the following formula.

$$
S i=\sum_{j=1}^{D} \operatorname{Min}\left(H a i^{j}, H b i^{j}\right) \text { divisionMin }\left(\sum_{j=1}^{D} H a i^{j}, H b i^{j}\right)
$$

Where, 
Hai and Hbi are the MLBP (multi scale local binary pattern) histogram that are extracted from Ai,

Bi respectively.

The similarity is matched by comparing a descriptor histogram for $\mathrm{R}=1,3,5$ and 7 for sketch image with corresponding the descriptor histogram for $\mathrm{R}=1,3,5$ and 7 for facial photo is compared with the help of this method we get 32196 histogram so, we have to find the mean of 32196 and that mean value is stored. Here, we have considered two types of similarity, the first one is perfect match which is obtained with the help of maximum value of histogram descriptors and second is nearest match which is obtained with the help of nearest value of histogram descriptors.

The CBR process is helping to match a composite sketch with facial photograph. Here, The sketch is considered as a query sketch and this sketch is compared with all the facial photographs that is stored in a database in our case here we have considered 30 composite AR database for female with corresponding a 20 facial photograph and the same is considered for 30 composite AR database for male with corresponding a 20 facial photograph .

\subsection{Contribution}

The MBLP is applied on each of $12 * 12$ window with $60 \%$ overlapping and finally a histogram descripotrs is obtained with the help of this we get 32196 histogram, but it is impossible to store 32196 histogram so we have to find "Mean" and on the basis of this we are calculating a similarity for the best match and nearest match, the best match is obtained with the help of maximum value of mean and nearest match is obtained with the help of nearest value of the mean.

\section{Experimental Results}

\subsection{Composite Sketch Database}

In our experiment we choose to use the AR database because it has been used in the previous study also. The composite sketches are constructed with the help of FACES and Identi Kit. Here we have 20 facial photographs and 20 composite sketches for female and same for male also which is obtained from AR database.

\subsection{Experimental Analysis}

Table1: shows the Gender distribution of Composite Sketches and Mug shot

\begin{tabular}{|l|l|l|l|l|l|}
\hline S. N. & Data Set & $\begin{array}{l}\text { Male } \\
\text { Images }\end{array}$ & $\begin{array}{l}\text { Male } \\
\% \\
\text { Accuracy }\end{array}$ & $\begin{array}{l}\text { Female } \\
\text { Images }\end{array}$ & $\begin{array}{l}\text { Female } \\
\% \\
\text { Accuracy }\end{array}$ \\
\hline $\mathbf{1}$ & $\begin{array}{l}\text { AR Composite \& } \\
\text { photographs }\end{array}$ & $\mathbf{9 0}$ & $\mathbf{6 4 \%}$ & $\mathbf{1 0 0}$ & $\mathbf{5 6 \%}$ \\
\hline
\end{tabular}


Table 2 Show a Similarity values of different sketch along with facial photographs In table 1, it shows the Gender distribution of composite sketches and mug shot, There they have consider a AR Composite and Photographs dataset having 90 male images and getting 64\% accuracy where as they have take 100 female images and getting 56\% accuracy. In this experiment table 2 shows the similarity values of different sketch along with facial photographs.

In table 3, it shows the experimental analysis on the basis of our "Contribution", here the same data set i.e AR composite and photographs are consider along with 90 male sketch with corresponding 80 facial photographs and getting $70 \%$ accuracy where as 100 female sketch is consider with corresponding 80 facial photographs and getting $68 \%$ accuracy. In this experiment no manipulation in the dataset is done.

Table 2: A Similarity values of different sketch

\begin{tabular}{|c|c|c|c|c|c|c|c|c|c|c|c|c|c|c|c|c|c|c|c|c|}
\hline & A & B & C & $D$ & $\mathrm{E}$ & $F$ & G & H & 1 & 1 & K & & M & 11 & 0 & & $Q$ & & s & 1 \\
\hline \multicolumn{3}{|c|}{1 similarity index } & \multicolumn{2}{|c|}{ similarity index } & \multicolumn{2}{|c|}{ simiarity index } & \multicolumn{2}{|c|}{ simialrity index } & \multicolumn{2}{|c|}{ similarity index } & \multicolumn{2}{|c|}{ similarity index } & \multicolumn{2}{|c|}{ similarity index } & \multicolumn{2}{|c|}{ similarity index } & \multicolumn{2}{|c|}{ similarity index } & \multicolumn{2}{|c|}{ similarity index } \\
\hline 2 & 0.516652 & 1 & 0.454105 & 2 & 0.524127 & 3 & 0.492781 & 4 & 0.454514 & 5 & 0.467243 & 1 & 0.420256 & 7 & 0.462171 & 3 & 0.470746 & & 0.443491 & 15 \\
\hline 3 & 0.488248 & 3 & 0.433338 & 8 & 0.454525 & 1 & .436621 & 19 & .42577 & 10 & 464305 & & 414554 & & 453957 & & 435537 & & 441102 & 10 \\
\hline 4 & 0.460623 & 19 & 0.415522 & 1 & 449945 & 15 & 33412 & 14 & 0.419788 & 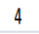 & 150901 & & 4999 & & 444212 & & 0.429562 & & 23268 & 13 \\
\hline 5 & 0.457455 & 4 & 13815 & 10 & 40149 & 7 & 8897 & 3 & 16341 & & 137739 & & 58556 & & 40889 & & 428718 & & & 7 \\
\hline 6 & 447245 & 6 & 10774 & 3 & 0.435731 & 19 & 15768 & 1 & 111800 & 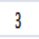 & 311146 & & 379627 & & 436448 & & & & 18117 & 14 \\
\hline 7 & 0.444077 & 12 & 03404 & 5 & 2991 & 18 & & 17 & 11289 & 15 & 29239 & & 78003 & 15 & 34622 & & 408803 & & 14704 & 19 \\
\hline 8 & 0.440958 & 15 & 0.401085 & 6 & 0.425806 & 17 & 0.420117 & 15 & 41007 & & 0.427476 & & 755994 & & 429451 & & 0.408458 & & 413033 & 16 \\
\hline g & 0.435142 & 14 & 195094 & 19 & 42396 & 16 & 114479 & 20 & 107044 & 1 & 122443 & $1 /$ & 65375 & & & & 406004 & & 08804 & 3 \\
\hline 10 & & 9 & & 15 & & 20 & & 7 & & & & & & & & & & & & 4 \\
\hline 11 & 0.429543 & 17 & 0.385927 & 4 & 0.416066 & 14 & & 6 & 0.402541 & & 0.418908 & & 0.365107 & & 0.41427 & 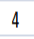 & 0.388735 & & 391039 & 1 \\
\hline 12 & 0.422153 & 18 & 0.384635 & 16 & 0.413035 & 4 & 400541 & 18 & 0.396571 & 6 & 0.416479 & & 363881 & & 406505 & 20 & 0.383294 & & 386595 & 17 \\
\hline 13 & 0.421837 & 7 & & 14 & & 6 & & 12 & & 10 & & & & & & & & & & 18 \\
\hline & 0.405712 & 11 & & 13 & & 12 & & . & & & & & & & & & & & & 8 \\
\hline 15 & 0.405166 & 8 & 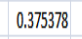 & 9 & 90977 & 13 & & 16 & & & & & & & 7.398139 & & & & & 6 \\
\hline 16 & 0.399696 & 13 & 0.372425 & 7 & 0.396498 & 9 & 0.3908 & 2 & 0.381734 & 13 & 0.399856 & & 0.356945 & & 0.397968 & 12 & 0.372242 & & 0.376665 & 11 \\
\hline 17 & 0.399505 & 20 & & 18 & & 11 & & 5 & & & & & & & & & & & & 20 \\
\hline 18 & 0.394331 & 16 & & 12 & & 8 & & 9 & & & & & & & & & & & & 5 \\
\hline 19 & 0.388043 & 2 & & 17 & 0.374313 & 10 & & 13 & 0.372681 & 2 & 0.387299 & 10 & 0.335071 & & 0.381683 & & 0.361002 & & 1363736 & 9 \\
\hline 20 & 0.380392 & 5 & & 11 & & 2 & & 8 & & & & & & & & & & & & 2 \\
\hline 21 & 0.370375 & & 00.352973 & 20 & 0.348652 & 5 & 0.374004 & 11 & 0.349652 & 12 & 0.374206 & & 0.323649 & 12 & 0.370396 & 2 & 0,346986 & 20 & 0.353018 & 12 \\
\hline
\end{tabular}

Table 3: Shows the experimental analysis on the basis of our Contribution.

\begin{tabular}{|l|l|l|l|l|l|}
\hline S.N. & Data Set & $\begin{array}{l}\text { Male } \\
\text { Images }\end{array}$ & $\begin{array}{l}\text { Male } \\
\% \text { Accuracy }\end{array}$ & $\begin{array}{l}\text { Female } \\
\text { Images }\end{array}$ & $\begin{array}{l}\text { Female } \\
\% \text { Accuracy }\end{array}$ \\
\hline 1 & $\begin{array}{l}\text { AR Composite } \\
\text { and Photographs }\end{array}$ & $\mathbf{9 0}$ & $\mathbf{7 0 \%}$ & $\mathbf{1 0 0}$ & $\mathbf{6 8 \%}$ \\
\hline
\end{tabular}

\subsection{Average Calculation of Face Recognition Accuracy}

Here, we have Consider 10 Female Composite Sketches (From AR dataset) Also we consider 20 Female Facial Photographs (From AR dataset)

1. The Similarity Calculation is shown in table 2. Form this, table we can concluded that sketch no. $5,6,8$ is not perfectly matched (it gives a nearest matched). 
2. Matched image $=($ no. of query sketch $)-($ Unmatched sketch with an image $)$ $=10-3=7$

3. In terms of percentage $=70 \%$

4. Accuracy Increase $=(70-43.1) \%=26.9 \%$

5. Accuracy increased $=27 \%$

\section{Results}

Here we try to match the facial photographs with the help of composite sketches with the help of CBR approaches we are getting a perfect matching.

\section{Conclusion}

The component based representation is an effective approach for matching a composite sketch to the facial photographs and it also measure the similarity between the composite sketches and the mug shot photographs. Hence the CBR approach will successfully recognize the face based on the sketches.

\section{Future Work}

Good intensity is used to remove the light illumination and more precise feature can be extracted from the face as well as sketches. The Automated technique can be used to extract the facial components, the face and sketch can be made intensity homogenous and also more similarity calculation factors can be added

\section{References}

[1] Arfika Nurhudatisna, Adams Wai Kin Kong “ On Criminal Identification In Colour Skin Images using Skin Marks(RPPVSM) and Fusion with Inferred Vein Patterns" IEEE Transactions On Information Forensics And Security, Vol. 10, Issue: 5, Page No. 916931, Year: 2015

[2] Han Su, Adams Wai Kin Kong" A study on Low Resolution Androgenic Hair Pattern for Criminal and Victim Identification" IEEE Transactions On Information Forensics And Security, Vol. 9, Issue: 4 , Page No: 666-680, Year: 2014

[3] Hu Han, Brendan F. Klare, Kathryn Bonnen, and Anil K. Jain. "Matching Composite Sketches to Face Photos: Component-Based Approach" IEEE Transactions On Information Forensics And Security, Vol. 8, Issue: 1, Page No: 191-204 ,January 2013

[4] Himanshu S. Bhatt, Samarth Bharadwaj, Richa Singh and Mayank Vatsa, " Memetically Optimized MCWLD for Matching Sketches With Digital Face Images" IEEE Transactions On Information Forensics And Security, Vol. 7, Issue:. 5, pp.1522-1535, 2012.

[5] B. Klare, Z. Li, and A. Jain, "Matching forensic sketches to mug shot photos," IEEE Trans. Pattern Anal. Mach. Intell., Vol. 33, Issue: 3, pp 639-646, Mar. 2011.

[6] H. Han, S. Shan, L. Qing, X. Chen, and W. Gao, "Lighting aware preprocessing for face recognition across varying illumination," in PROC.EUR. CONF. Computer Vision, 2010, pp. 308-321.

[7] Yong Zhang, Member, Christine McCullough, John R. Sullins, Member and Christine R. Ross "Hand-Drawn Face Sketch Recognition by Humans and a PCA-Based Algorithm for Forensic Applications" IEEE Transactions On Systems, Man, Ancybernetics-Part A: Systems And Humans, Vol. 40, Issue:. 3, Page No: 475-485, May 2010. 